\title{
Commentary
}

\section{New Light on Chromotherapy: Grakov's ‘Virtual Scanning' System of Medical Assessment and Treatment}

\author{
Alex Hankey ${ }^{1}$ and Elena Ewing ${ }^{2}$ \\ ${ }^{1}$ Hethe House, Hartfield Road, Cowden, Edenbridge, Kent TN8 7DZ; ${ }^{2}$ Mulberry House, Vine Farm Close, Cotgrave, \\ Notts NG12 3TU, UK
}

\begin{abstract}
Virtual Scanning incorporates novel uses of colored light into its system of health assessment and therapy. Independent investigations of its effectiveness in Russia and the UK have revealed unique abilities to correct incipient and fully developed chronic conditions. As such it forms an important new addition to the field of Chromotherapy. It differs from most others, in that its development depended on discoveries in neuroscience by its inventor, and subsequent application of new models in computational neuroscience.
\end{abstract}

Keywords: chromotherapy - computational neuroscience - virtual scanning

\section{Introduction}

In a recent article in $e C A M(1)$, Azeemi and Raza reviewed the field of Chromotherapy, providing a history of the subject since ancient days (2), and its use in modern times (3). In the past 8 years, Virtual Scanning, a new Russian system of medical assessment and treatment, has revolutionized the use of precise light frequencies in the visible spectrum for preventive treatment, and where possible cure, of specific pathologies. The new system is still little known in countries outside the former Soviet Union, explaining its omission from Azzeemi and Rasa's review (1). Such is Virtual Scanning's revolutionary significance that no review of color therapy could be considered complete without describing it.

The fundamental influence of light in stimulating organismic function, and thus health, is well known (4), but modern medical science mostly attributes this to its effect on the pineal (5), since the hormones of that 'master gland' regulate pituitary and endocrine function and are thus necessary for the healthy functioning of the whole organism, yielding a safe, reductionist model for the beneficial influence of light on health. More subtle forms of Chromotherapy reviewed by Azeemi and Raza (1) hold that different colors influence organismic function differently, so that specific colors may benefit particular pathological

For reprints and all correspondence: Alex Hankey, Hethe House,

Hartfield Road, Cowden, Edenbridge, Kent TN8 7DZ, UK.

Tel: +44-1342-85-00-86; E-mail: alexhank@dircon.co.uk conditions. Such forms of Chromotherapy may depend on the influence of light on the system of nadis/acumeridians; they lack known mechanisms, and belong to CAM.

Azeemi and Rasa attempt to remedy this lack of mechanism, making their work of interest to those seeking biologies of CAM $(6,7)$. To understand how different colors may differently influence organismic function, they hypothesize that colored light may be able to influence the internal structure of water. Should they be correct, their hypothesis links to recent suggestions by Tiller et al. (8), that so-called 'water-memory' originates in the internal structural arrangements of its hydrogenbond linked oxygen atoms. The hypothesis offers insight as to how different colors may possess different 'vibrational' properties, by which they could influence health through principles of vibrational medicine linked to homeopathy $(9,10)$. It would also link to Emoto's structures of water crystals (11). The significance of Virtual Scanning to this debate is that it represents a completely new, medical technology of light, based on discoveries of new mechanisms and neural pathways, by which light may influence organ and organismic function.

\section{Grakov's 'Virtual Scanner’}

Virtual Scanning is an empirically established system of health assessment and treatment (12), using specific effects of organ function on color cognition, and color stimulation on organ health. It is based on principles discovered at the University of Krasnoyarsk by Professor Igor Grakov (13), who subsequently 
developed his system into its present form (14). It was evaluated at the University of St Petersburg by Vysochin (15), and at other medical centers (16). On the basis of a 10-15 min color cognition test taken on a computer screen, Virtual Scanning yields an assessment of the health of 30 organs (of which 3 are sex-specific), an amazing achievement backed up by clearly delineated concepts, well observed case studies (12), and considerable research data (15).

Grakov discovered how the structure of cortical information processing and analysis, permits systematic errors in color cognition to be used for accurate health assessment; and conversely, how administering precise sequences of colors, at precise frequencies of repetition, for given periods of time, can stimulate healing in designated organs.

More precisely, the discoveries that enabled Grakov to develop his system were as follows:

(i) Organs transmit precise information concerning their functional state to the brain.

(ii) The information from each organ precisely expresses the degree of its degeneration towards various kinds of pathology_different sets of pathologies for each organ.

(iii) For each such pathology developing in any organ, the brain has the capacity to transmit neural signals to stimulate the organ, precisely compensating for any loss of function caused by the pathology, thus restoring organ function to normal.

(iv) The brain stores information about all such signals: (i) concerning organ pathologies and (ii) concerning their compensation; in such a way that

(v) Each signal gives rise to its own specific, identifiable influence on color cognition.

This means that particular pathologies in particular organs, together with the degree to which each is being compensated, influence brain color processing in precise, distinguishably different, ways. Grakov's genius was to design, first, a test measuring deviations from normal color cognition, which can assess the magnitude of all these processes, and second, a therapy using related brain circuits to direct precise, corrective, healing influences to the brain and organs concerned for each condition detected.

Thus, the Virtual Scanning Health Assessment test uses the influence of the brain's own information processing on color cognition to measure the following:

(i) Health information transmitted from each organ to the brain, and

(ii) The amount the brain is stimulating each organ to compensate for such problems.

Virtual Scanning's system of treatment uses correspondingly selected colors delivered at specific frequencies of repetition in the delta band of EEG, used by the brain to direct healing to the organs during the night. Hence the name, 'Virtual Scanning': the system uses the virtual reality of the brain's information processing, to provide both body scan and remedial treatment.

\section{Case Histories}

To most people, the idea that precisely selected colors can help specific pathologies, on occasion producing remarkable cures, may sound unlikely. However, the following case histories from the UK and Russia, show the system can produce remarkable results:

\section{Assessment}

(i) A UK businesswoman of 47 with her own company had reported upper abdominal discomfort to her GP. She had also been taking aspirin for a year as a secondary prevention measure against platelet aggregation. The GP had not detected any identifiable pathology, and suggested the cause was stress. Virtual Scanning Health Assessment detected a developing ulcerative process in the duodenum, requiring urgent treatment. This was declined by the patient as was a suggestion to stop the aspirin. She returned to her GP who reiterated his opinion, suggesting a good rest and holiday. Within a week she was admitted to hospital for 10 days with upper gastrointestinal bleeding diagnosed as a duodenal ulcer.

(ii) Assessment of a man of 57 in the UK, suffering from bouts of disorientation, revealed inter alia vertebral artery syndrome, poor blood flow to the back of the brain and undue pressure on the cervical vertebrae. It was determined that the pressure on the head bones was being caused by overexertion on Hala Asana, the Yoga position known as 'The Plough', suggesting that this was causing overstretching of the arteries in the neck (as well as the muscle tendons the posture is intended to stimulate and rebalance). On stopping all such Yoga postures, the feelings of giddiness and disorientation went away within 2 weeks. Retesting showed both pressure on the head bones and the restricted blood flow to have been eliminated.

\section{Treatment}

(i) A Russian epileptic, who had been invalided from age 5 , suffered up to 10 attacks per day in spite of taking all prescribed medication. She also suffered from headaches. Virtual Scanning diagnosis led to a prescription of Virtual Scanning therapy with the brain module. After her first 21 day course of treatment all attacks ceased, and she was able to stop taking any medication. Some time later, she was given a further preventative course of Virtual Scanning treatment, after which no further attacks were reported. Clinical investigation showed that the activity in the cortex responsible for the attacks had disappeared. The patient ceased to be categorized as disabled.

(ii) Assessment of a 59-year-old woman, who had suffered migraine attacks from age 11, worsening in recent years, confirmed migraine and also indicated vertebral 
artery syndrome, impaired spinal blood flow, osteochondropathy with neurological effects and idiopathic hypotension (Fig. 1), for all of which treatment was prescribed.

One week prior to the test, the patient had been admitted to hospital fainting from severe pain, and given seven different medications in a $6 \mathrm{~h}$ period. After 10 months of Virtual Scanning treatment, she had not suffered a single further migraine attack. Assessments following successive periods of treatment indicated the underlying tendencies causing her migraine to be slowly reducing.

The significance of these case histories is not just that the health assessment was able to confirm previous diagnoses, but that it was also able to identify underlying causes. Together these imply that Grakov's basic theory must be correct, constituting a series of important discoveries about (i) the structure and function of neural signals between the brain and peripheral organs and (ii) the brain's mechanisms of data processing.

\section{The virtual Scanning Model}

Central to Grakov's theory is a two matrix model of how organ information influences cognition: an internal matrix $\boldsymbol{M}_{\mathbf{i}}$ recording information received from organs and organ systems, and an external matrix $\boldsymbol{M}_{\mathbf{e}}$ monitoring the external environment, in constant communication with each other. The two matrices, together with their respective communication flows, are schematically depicted in Fig. 2.

Apparently, similar biomathematical models apply to most, if not all, sensory channels. Grakov has applied it to others in addition to sight. In the case of color processing and cognition, the model is normalized for the weight, age and sex of each subject. Its algorithms analyze colors in a grid, the grid point parameters being modified according to the signals transmitted to and from the brain and recorded in the internal matrix. Changes in organ health shift the grid parameters, so that changes occur in color cognition, reflected by specific alterations in color test performance. In health, test results indicate 'normal' color processing (according to sex, age and weight). For each organ, specific deviations from health result in identifiable changes in test performance.

According to Grakov, deviations from normal health in any organ result in information flows to the brain specific to each kind of deviation. His work indicates that such signals are present long before detectable pathology manifests, and that each kind of signal will increase to a point where a specific pathology becomes detectable. Such pathologies are used to label signal types: if, as a particular signal from, e.g. the liver (with a dozen or more signal types) increases, neoplasm becomes detectable, then 'neoplasm' is used to label the signal; similarly for the signal labeled 'chronic fatigue' etc.

\section{Discussion}

Virtual Scanning assessments and treatments could not begin to work unless the Internal-External Matrix model of sensory information processing was correct: success rates reported by Vysochin (15) are far higher than any placebo investigated. Surprising though it may seem, Grakov's model must basically be valid. The only questions are ones of degree: degree of accuracy of the system's medical assessments, and, when properly administered, degree of success of its treatments.

One major implication of Grakov's work is that each organ transmits as many kinds of distinguishable neural signals to the brain as the different kinds of ailment his system can detect for the organ in question, i.e. approximately 15 different signal types related to the state of health of each organ. How neural signals encode such information is an important question requiring experimental elucidation.

The next implication is that the analytical processing identified by Grakov must take place in the physiology. The question is where? A simple hypothesis is that it takes place in the cortex. This is for two reasons: first, the cortex contains structures able to retain the information designated for the internal and external matrices; second, different layers of the cortex are interconnected in such a way that the required mathematical processing could be carried out (the cortex is comprised of six separate layers, each with its own function and interconnections to other layers). Grakov's neural model has implications for (i) the function of the appropriate layers, and (ii) the way different levels of the cortex interact with each other. Regions of cortex storing sensory input are possible locations for the external matrix $\boldsymbol{M}_{\mathbf{e}}$, while regions storing somatic information may be locations for the internal matrix $\boldsymbol{M}_{\mathbf{i}}$.

The length of the interconnections, with dendrites spreading considerable distances, make the relationship of the activity of one cortical region to that of another a uniquely interesting mathematical transformation. The linear transforms between cortical layers implied by Grakov's matrices and neural models would effectively enable the whole cortex to function in a holistic fashion. This is of great importance to complementary medicine, for it implies that higher organisms function holistically, providing support for the concept of a 'holistic biology', underlying 'holistic medicine'.

A final implication of Grakov's work is that some form of natural color therapy would have been possible in the wild. This is because improvements in organ condition result in improved sense of well-being. Thus a creature's attention would be specifically drawn to a color with remedial qualities for any problem in its organs, since looking at that color would produce a natural positive feedback. Such a possibility is also the case for ordinary color therapy. On sunny days in spring time on the streets of Stockholm and other Nordic cities, citizens can traditionally be seen resting with their face in the sunshine for quite lengthy time periods, not because they may have been medically advised to do so, but because it makes them feel good-they are activating the pineal after all the darkness of the winter months, and consequent melatonin deprivation.

Grakov's model implies that any creature, which developed the neurological functions and connections required, would 
(a)

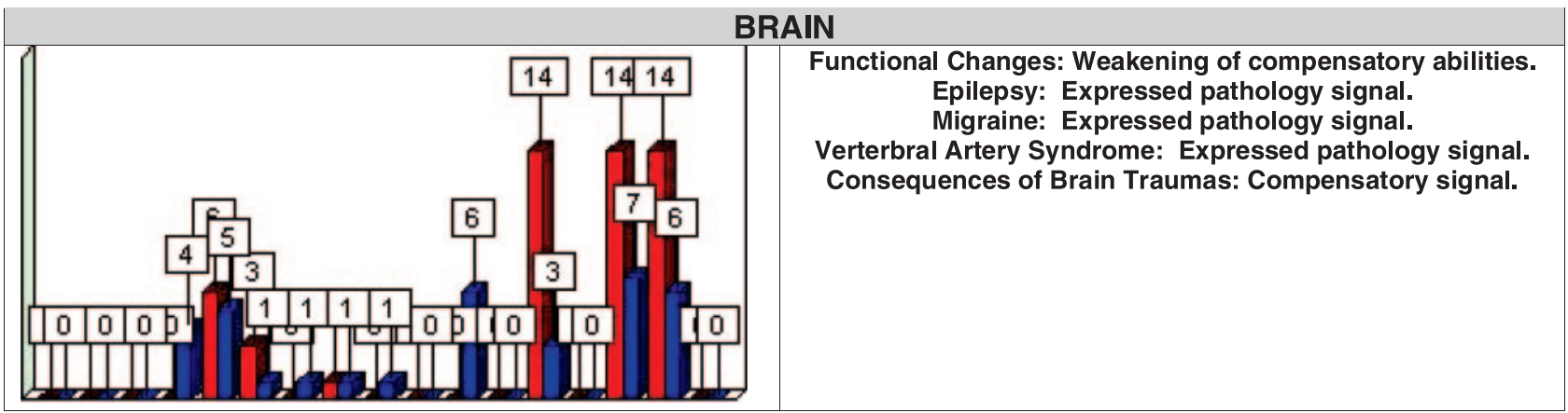

(b)

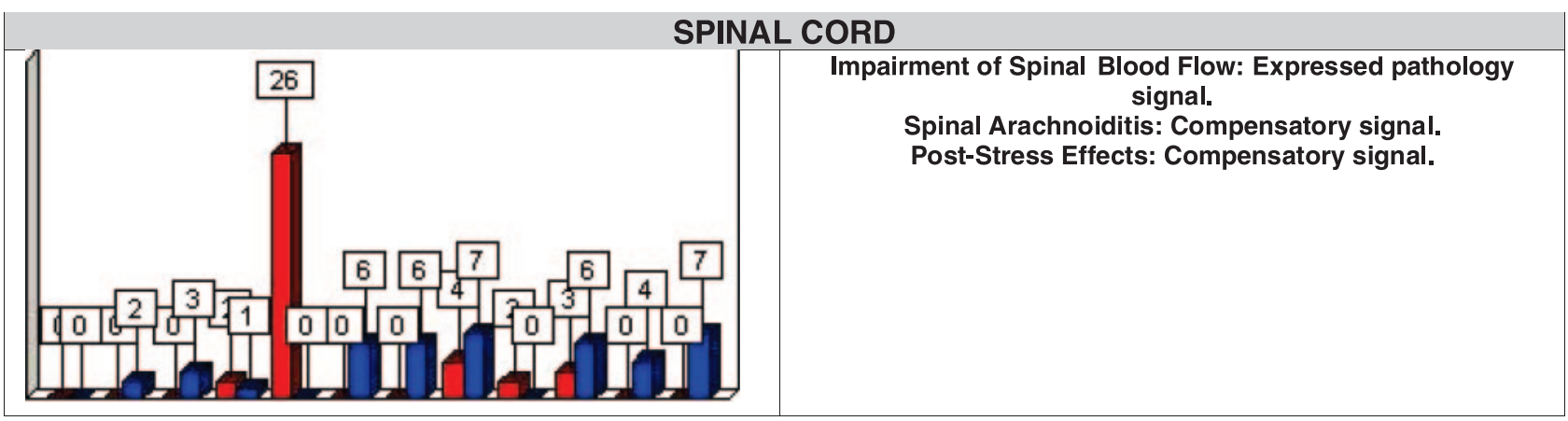

(c)

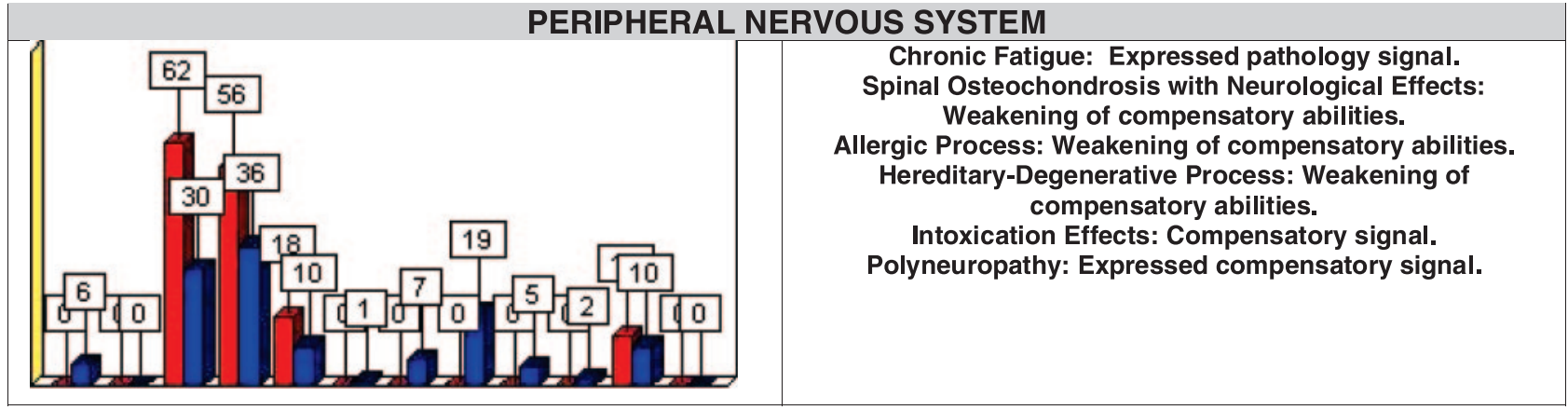

(d)

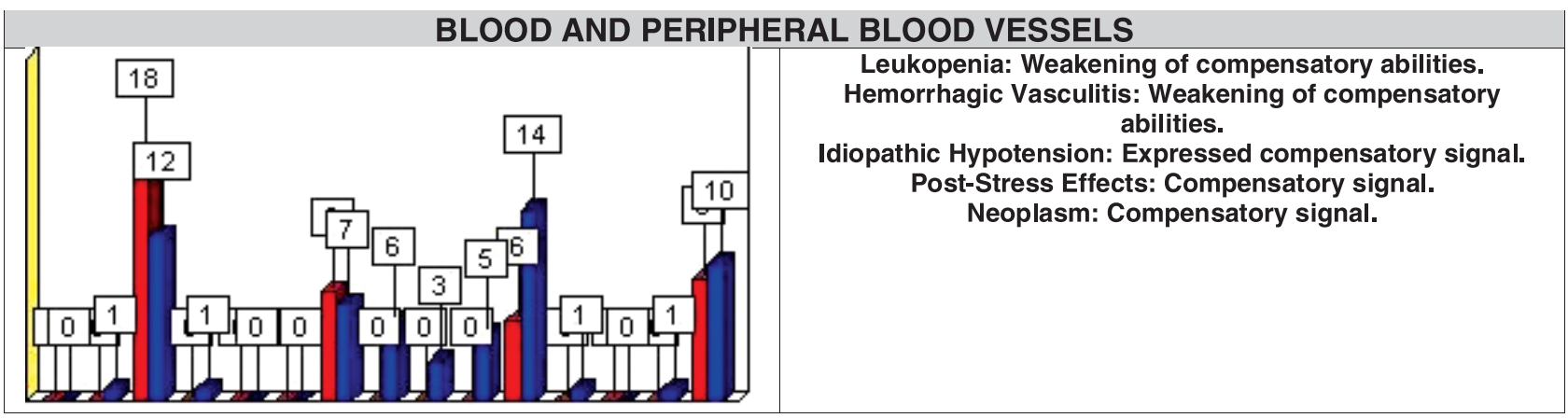

Figure 1. (Case history Treatment (ii): 59-year-old patient with migraine.) This example illustrates how Virtual Scanning can yield an overall picture of a patient's pathology, including its underlying causes. Here, signals from brain (A), spinal chord (B), peripheral nervous system (C) and blood (D) show indications of uncompensated vertebral artery syndrome (A), impaired spinal circulation (B), osteochondropathy with neurological effects (C) and idiopathic hypotension (D). Together these form a connected set of underlying causes for the pathology: the osteochondropathy (C) can lead to the vertebral artery syndrome (A), which in turn leads to impaired spinal blood flow (B) and so to the ideopathic hypotension (D). Conclusion: these associated pathologies form the probable causes of the migraine. (A) Brain: note the migraine signal (14/7), and other indications of problems in brain function. The signal for vertebral artery syndrome [signals (14/6)], in particular, is often seen as one of the possible causes for migraine. (B) Spinal cord: the signals for uncompensated impairment of spinal circulation (26/0) show that the osteochondrosis (C) and consequent vertebral artery syndrome (A) connect into a specific pattern of causes of the migraine. (C) Peripheral nervous system: the poorly compensated osteochondrosis signal (56/36) indicates a probable cause for the vertebral artery syndrome signal in (A). (D) Blood and peripheral blood vessels: compensatory signals for idiopathic hypotension (6/14), are often seen in migraine cases, particularly when circulation problems are present [see (B)]. 


\section{EXTERNAL ENVIRONMENT}

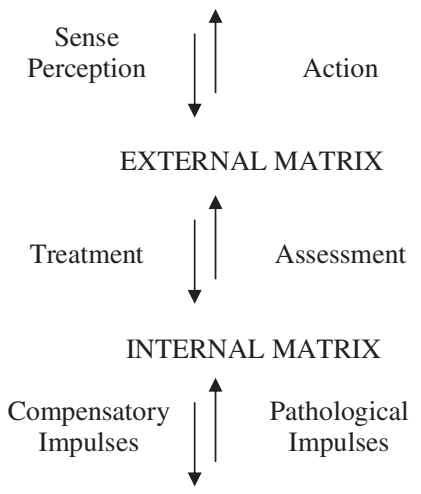

INTERNAL ENVIRONMENT

Figure 2. Schematic representation of Grakov's model. Figure 2 depicts Grakov's internal and external matrices in communication with the organism's internal and external environments, and with each other. The internal matrix receives information termed 'pathological impulses' from internal organs concerning deviations from normal, healthy function. It is responsible for 'compensatory impulses' sent back to compensate for such dysfunction. It also transmits information to the external matrix concerning the levels of both sets of impulses. The external matrix receives information from the external environment and the internal matrix. It is responsible for both cognitive processing and behavior (action). Information about levels of pathological and compensatory impulses received from the internal matrix modifies cognitive processing of sense perception data received from the external environment in precise ways. This is the basis for Virtual Scanning assessment. Conversely, specific sense perception data received by the external matrix can be made to stimulate the internal matrix to modify its levels of compensation, and to send healing impulses to the organs. This is the basis for Virtual Scanning treatment-its specific form of Chromotherapy, made precisely according to its assessment.

gain a competitive advantage: the possibility of naturally maintaining a higher level of health. In support of this, consider comparable examples of instinctive reactions leading to the identification of curative or health promoting materials:

(i) Domestic animals tend to eat plants of various kinds when they are sick, particularly grass—not part of their normal, carnivorous diet;

(ii) During pregnancy, females of a species may seek out unusual materials (again, not their habitual foodstuffs) rich in materials required by the fetus at that stage of development, e.g. calcium or other minerals, when bones and blood are forming;

(iii) Edward Bach recorded that, in his discovery of the flower remedies named after him, he would begin to suffer from the condition, which a particular flower could assist, and, based on his 'feeling' response, would identify the flower in question.

These examples show that instinct leads various kinds of mammal, including humans, to seek out appropriate nourishment, both for special normal conditions, and to counteract pathology. There is every reason to believe that, although uncommon in this day and age, such abilities are part of the range of human abilities among the population ensuring long-term species survival-that is to say that they are commonly present among a steady fraction of members of the species.

The examples suggest that if organ health can be improved by experiencing particular stimuli, as Grakov has demonstrated, that is good reason for physiological properties and structures promoting it to have developed naturally: the system has a valid biology $(6,7)$. Reasons given above suggest that the structures include the brain cortex with all its layers for data processing, transformation and cognition. Could such possibilities for improved organ health and function have actually stimulated cortical development?

\section{Summary}

Although the fundamental influence of light in stimulating organismic function, and thus health, are well known, most medical thinking attributes this to its effect on the pineal, pituitary and neuroendocrine system. Chromotherapy is still a relatively obscure aspect of complementary medicine, particularly in the subtle forms reviewed by Azeemi and Raza (1). Although their hypothesis that colored light may be able to influence the internal structure of water may have substance, Grakov's account of his system of Virtual Scanning offers another way to understand the influence of color on health: the central nervous system is so constructed that there is a feedback between color cognition and organ monitoring and stimulation by the brain. When an organ is in the process of slowly developing a pathology, it emits increasingly strong neural signals to the brain indicating the degree of degeneration of its condition; the brain responds by applying appropriate compensatory stimulation. Color processing is among many aspects of CNS function influenced in specific ways by all such signals, with the following results:

(i) Color tests can be used to identify both organ pathology and brain compensation. (ii) Stimulation by appropriate colored light has a therapeutic effect on specific organs.

Specifically, Grakov's Virtual Scanning test of color cognition yields values for the following:

(i) Health information transmitted to the brain from each organ.

(ii) Neural signals transmitted by the brain to stimulate each organ and compensate for problems detected.

Successful applications of Grakov's system provide empirical support for his proposals. The latter offer insights to understand other phenomena in different fields of biology and the health sciences, for example, how known benefits of meditation practice may benefit health $(17,18)$, or, in sense perception, the problem of the so-called anosmias, where a person with an otherwise well-functioning sense of smell may be completely unable to detect the aroma of particular molecules (19)

Virtual Scanning is well established in Russia, where it has been found to yield valuable results for many pathologies. Many good case studies are emerging in the west. Since the 
mechanisms it proposes for the influence of different colored light on specific health problems lie within the structure of conventional science, Virtual Scanning deserves consideration by biomedicine and CAM, as well as those interested in chromotherapy.

\section{Acknowledgments}

We would like to acknowledge conversations with Graham Ewing. I would also like to thank Harry Pilcher MSc, and the University of Pune, whose combined generosity made this work possible.

\section{References}

1. Azeemi ST, Raza SM. A critical analysis of chromotherapy and its scientific evolution. Evid Based Complement Alternat Med 2005;2:481-8.

2. Coclivo A. Coloured light therapy: overview of its history, theory, recent developments and clinical applications combined with acupuncture. Am J Acupunct 1999;27:71-83.

3. Azeemi S. Color Therapy. Karachi: Al-Kitab Publications, 1999.

4. Breiling B. Light Years Ahead: The illustrated Guide to Full Spectrum and Colored Light in Mind/Body Healing. Berkeley, CA: Celestial Arts, 1996.

5. Available at: http://en.wikipedia.org/wiki/Pineal_Gland.

6. Cooper EL. Complementary and alternative medicine, when rigorous can be science. Evid Based Complement Alternat Med 2004;1:1-5.

7. Hankey A. CAM modalities can stimulate advances in theoretical biology. Evid Based Complement Alternat Med 2005;2:5-12.
8. Roy R, Tiller WA, Bell I, Hoover MR. The structure of liquid water; novel insights from materials research; potential relevance to homeopathy. Mater Res Innov 2005;9:93-124.

9. Gerber R. Vibrational Medicine. Rochester, Vermont: Bear \& Co., 2001.

10. Hankey A. Are we close to a theory of energy medicine? J Altern Complement Med 2004;10:83-6.

11. Emoto M. Message from Water. London: Hay House, 2006.

12. Ewing G, Ewing E, Hankey A. Virtual Scanning: a new method of health assessment and treatment. Part I: assessment. J Altern Comp Med 2006 (in press).

13. Grakov I. Strannik Diagnostic and Treatment System; a Virtual Scanner for the Health Service. Minutes of Meeting No. 11 of the Presidium of the Siberian of the Academy of Medical Sciences of the USSR (AMN) held in Novosibirsk 4 December 1985.

14. Grakov I. Description of Virtual Scanning System for Operators. Mimex, Sochi, Russia 2002, English translation available at: http://www. montague-diagnostics.co.uk/files/Grakov/Article7.pdf.

15. Vysochin Y. Methodology and Technology of Invigoration of Different Population Orders. In: Consolidated 5 year Research Plan of Physical Training, Sports and Tourism State Committee of the Russian Federation. 2000, English translation available at: http://www.montague-diagnostics. co.uk/files/Vysochin/Vysochin.pdf.

16. Skvortsova NP, et al. Internal Report on Virtual Scanner from Medical Department of Moscow Mint, National Enterprise. 1999.

17. Hankey A. CAM and post traumatic stress disorder. Evid Based Complement Alternat Med. Advanced Access published 6 July 2006.

18. Hankey A. Studies of advanced meditation in the Tibetan Buddhist and Vedic Traditions. I: A comparison of general changes. Evid Based Complement Alternat Med. doi:10.1093/ecam/nel040.

19. Lawless HT. Olfactory psychophysics. In: Beauchamp GK, Bartoshuk L (eds). Tasting and Smelling. Chapter 4, San Diego: Academic Press, 125-74.

Received July 10, 2006; accepted August 10, 2006 


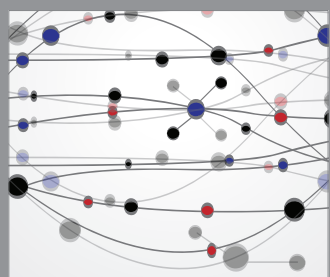

The Scientific World Journal
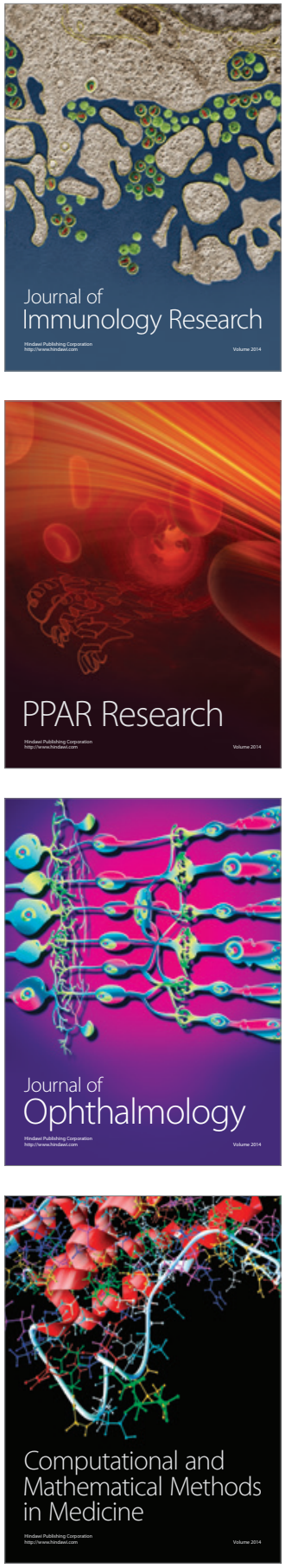

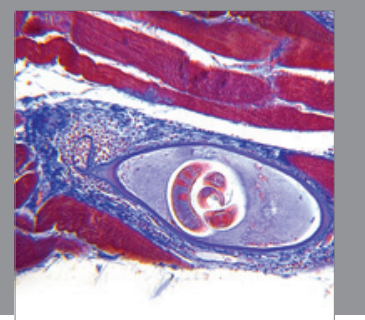

Gastroenterology

Research and Practice
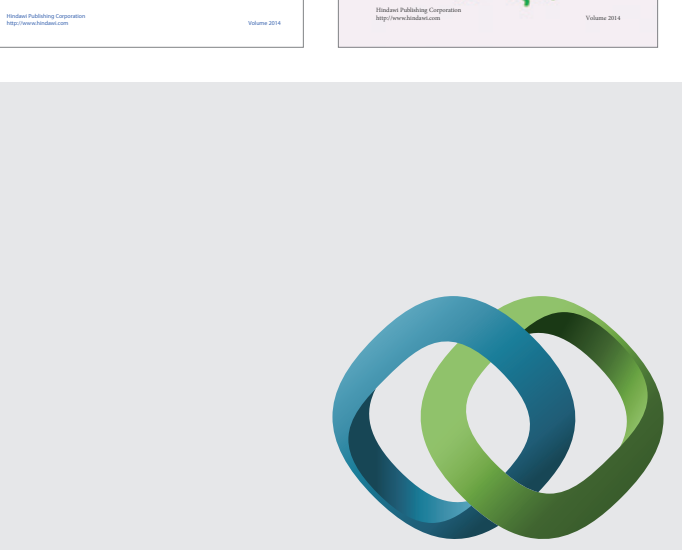

\section{Hindawi}

Submit your manuscripts at

http://www.hindawi.com
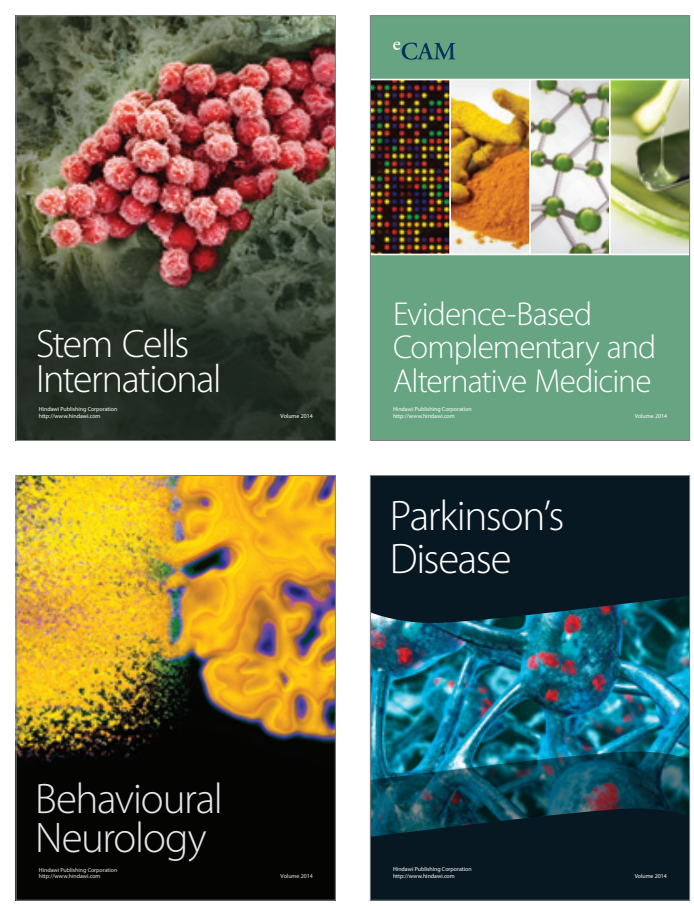

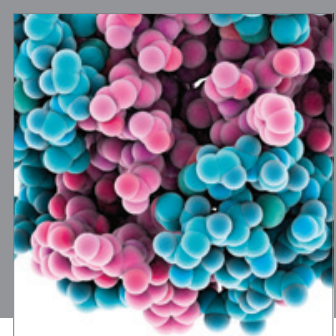

Journal of
Diabetes Research

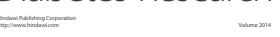

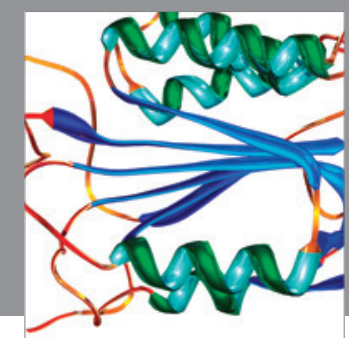

Disease Markers
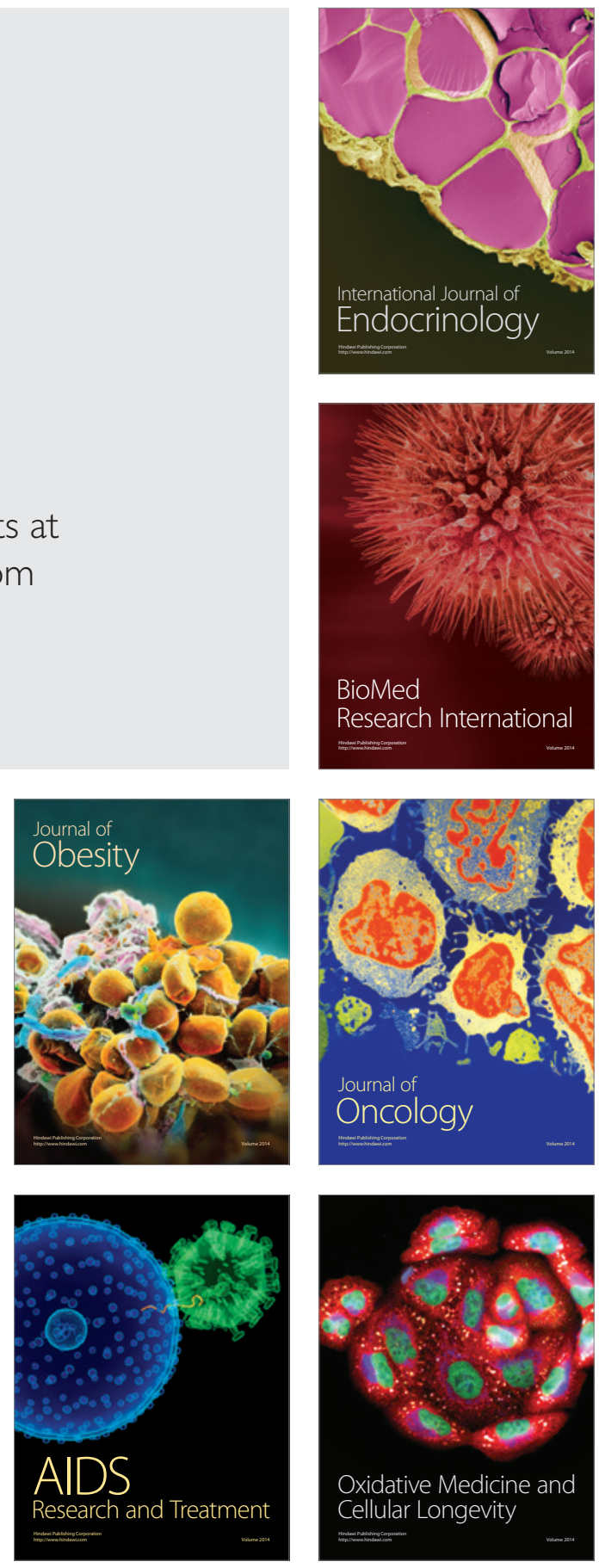